\title{
Mast cell stabilizer ketotifen reduces hyperalgesia in a rodent model of surgically induced endometriosis
}

This article was published in the following Dove Press journal: Journal of Pain Research

\author{
Tian-Hong Zhu' \\ Gen Zou' \\ Shao-jie Ding' \\ Tian-Tian $\mathrm{Li}^{\prime}$ \\ Li-Bo Zhu' \\ Jian-Zhang Wang' \\ Yong-Xing $\mathrm{Yao}^{2}$ \\ Xin-Mei Zhang' \\ 'Department of Obstetrics and \\ Gynecology, Women's Hospital, Zhejiang \\ University School of Medicine, Hangzhou, \\ People's Republic of China; ${ }^{2}$ Department \\ of Anesthesia, First Affiliated Hospital, \\ Zhejiang University School of Medicine, \\ Hangzhou, People's Republic of China
}

Correspondence: Xin-Mei Zhang Department of Obstetrics and

Gynecology, Women's Hospital, Zhejiang University School of Medicine, I Xue Shi Road, Hangzhou, Zhejiang 310006,

People's Republic of China

Email zhangxinm@zju.edu.cn
Purpose: This study aimed to investigate the effect of oral treatment with ketotifen, a mast cell (MC) stabilizer, in a rat model of surgically induced endometriosis.

Methods: At 14 days after Sprague-Dawley rats had surgery, they were treated with ketotifen ( 1 or $10 \mathrm{mg} / \mathrm{kg} /$ day). Pain behaviors were evaluated 3 days prior to surgery and then at $7,14,21$, and 28 days after surgery. At day 28 , rats were sacrificed and all samples were then processed for biochemical studies.

Results: We found that ketotifen-treated rats showed significantly shorter duration of hyperalgesia $(p<0.05)$; smaller cyst diameter $(p<0.05)$ and lower histopathologic score $(p<0.001)$; significantly lower MC number and degranulation $(p<0.001)$, blood vessel number $(p<0.001)$, lower expression levels of nerve growth factor $(p<0.001)$, cyclooxygenase-2 $(p<0.001)$, intercellular cell adhesion molecule-1 $(p<0.001)$, and vascular endothelial growth factor $(p<0.05)$ in cysts, and nerve growth factor $(p<0.001)$ and transient receptor potential cation channel, subfamily V, member $1(p<0.001)$ in dorsal root ganglia; and lower histamine $(p<0.05)$ and tumor necrosis factor-alpha $(p<0.05)$ concentrations in serum compared with placebo-treated animal subjects.

Conclusion: Oral treatment with ketotifen significantly suppressed the development of hyperalgesia, probably by modulating MC activity in cysts, thereby reducing peripheral sensitization due to noxious signals from endometriotic lesions. Our results suggest that ketotifen may inhibit the development of endometriotic lesions and hyperalgesia in rats.

Keywords: endometriosis, ketotifen, rat, mast cells, hyperalgesia

\section{Introduction}

Endometriosis is characterized by the presence of endometrial glands and stroma outside the uterine cavity. ${ }^{1}$ In women with endometriosis-associated pain, several treatments are available including nonsteroidal anti-inflammatory drugs (NSAIDs), oral contraceptives, gonadotropin-releasing hormone (GnRH) agonists, and surgical treatment. ${ }^{2}$ NSAIDs have significant side effects, including gastric ulceration ${ }^{3}$ and anti-ovulatory effects, ${ }^{4}$ when taken at mid-cycle. Meanwhile, menopausal side effects associated with GnRH agonists, such as osteoporosis, make them suitable only as short-term solutions. ${ }^{5}$ Temporary relief may be provided by laparoscopy and fulguration, or excision of endometriosis, but these treatment modalities often have a high recurrence rate. Therefore, a mechanism-based alternative treatment is necessary to control the symptoms of endometriosis. 
The number of mast cells (MCs) and degranulated MCs increases in endometriotic lesions. ${ }^{6-10}$ Endometriosis-induced pain is a type of inflammatory and neuropathic pain, ${ }^{11}$ and mediators such as histamine ${ }^{11-13}$ and tumor necrosis factor $(\mathrm{TNF}-\alpha)^{14}$ released by activated MCs contribute directly to neuropathic symptoms. In addition, activated MCs may also exert indirect effects on the development of neuropathic pain by recruiting leukocytes. ${ }^{15}$ Human endometriotic lesions show significantly higher numbers of activated MCs, located less than $25 \mu \mathrm{m}$ from nerve structures, than unaffected tissues. ${ }^{6}$ Although the role of MCs in the mediation of nociceptive signaling needs to be explored further, MC-released products in endometriosis can contribute to the development of pain and hyperalgesia by influencing neurons. Therefore, inhibiting $\mathrm{MC}$ function constitutes a new option in the treatment of this disease and its associated pain.

Degranulation of MC is blocked by membrane stabilizers such as chromones and ketotifen. In our previous study, the number of activated MCs and concentration of TNF- $\alpha$ in rat serum decreased considerably when the experimental group was treated with sodium cromoglycate. ${ }^{16}$ Thus, treatments aimed at stabilizing MCs may play a role in preventing endometriosis or relieving corresponding symptoms. Ketotifen has a stronger effect on stabilizing MCs than sodium cromoglycate. ${ }^{17}$ This drug has antihistamine activity ${ }^{18}$ and is also a functional leukotriene antagonist. ${ }^{19}$ It is mainly used in patients with asthma and other allergic diseases like allergic rhinitis and chronic urticaria; ${ }^{20}$ however, it has also been indicated to have anti-nociceptive and antiinflammatory effects in many pathological conditions, such as irritable bowel syndrome, ${ }^{21,22}$ fibromyalgia, ${ }^{23,24}$ and postoperative hyperalgesia. ${ }^{25,26}$ Although ketotifen causes adverse reactions such as drowsiness, dry mouth, and gastrointestinal discomfort, the incidence rate is generally low and such reactions only appear early in the treatment period. Thus, ketotifen is an ideal candidate for endometriosis treatment.

In this study, we aimed to investigate the antinociceptive and anti-inflammatory effects of ketotifen in the treatment of endometriosis in an experimental rat model by evaluating nociceptive behavior and biochemical and histopathologic parameters.

\section{Materials and methods}

\section{Animals}

Female Sprague-Dawley rats (weight: 220-260 g) were used for the study. Animals were housed in groups of six at $21 \pm 0.5^{\circ} \mathrm{C}$ under a $12 \mathrm{~h} \mathrm{light/dark} \mathrm{cycle} \mathrm{with} \mathrm{free} \mathrm{access} \mathrm{to}$ food and water. This study was carried out in strict accordance with the recommendations of the Guide for the Care and Use of Laboratory Animals of the National Institutes of Health. The protocol was approved by the Committee on the Ethics of Animal Experiments of Zhejiang University.

\section{Induction of experimental endometriosis}

Endometriosis was induced using autologous uterine horn transplantation based on established procedures. ${ }^{27}$ All rats were anesthetized with isoflurane, and the left uterine horn was excised. This tissue fragment was placed in phosphate buffer saline (PBS) at $37^{\circ} \mathrm{C}$ and cut along the longitudinal axis, obtaining four pieces of $3 \mathrm{~mm} \times 3 \mathrm{~mm}$ tissues. Two of the squares were sutured to the left and right sides of the inner surface of the abdominal wall, and the other two were sutured to the bowel mesentery close to a large vessel. All implants were sutured using a sterile $6-0$ silk suture, and the abdominal cavity was closed with a $3-0$ silk suture. The sham operation group was induced by removing the left uterine horn, without suturing it back to the abdominal wall or mesentery. Penicillin $(40,000 \mathrm{U} / \mathrm{kg})$ was administered for 5 days after the surgery.

\section{Experimental groups}

The sham operation group $(\mathrm{n}=6)$ was used as blank comparison. The remaining rats were randomly divided into three groups. Vehicle (carboxymethylcellulose sodium $0.5 \% \mathrm{w} / \mathrm{v}$ in saline, CMC-Na) was administered by oral gavage to the control group $(\mathrm{n}=6)$. Ketotifen was dissolved in CMC-Na and divided into the low dose group $(1 \mathrm{mg} / \mathrm{kg} /$ day, $\mathrm{n}=6)$ and high dose group $(10 \mathrm{mg} / \mathrm{kg} /$ day, $\mathrm{n}=6)$. The ketotifen dosage was based on previous data from the literature about the effects of ketotifen on pain behavior of other animal models. ${ }^{28,29}$ All the drugs were administered by oral gavage on the 14th day and for the next 14 days. To evaluate the effect of treatment, rats were sacrificed at 28 days after endometriosis induction. ${ }^{30}$ Rats were anesthetized with isoflurane, and laparotomy was performed to collect the endometriotic implants, dorsal root ganglia (DRG; T11-L2 segment), and serum from the inferior vena cava. All samples were then processed for biochemical studies.

\section{Nociceptive testing}

The animals were first acclimatized before values of the mechanical thresholds (MTs) and thermal latencies were measured. Measurements were made 3 days prior to 
surgery and then at $7,14,21$, and 28 days after surgery. The operator was unaware of the treatments that the animals had received.

\section{Mechanical hyperalgesia}

In response to mechanical stimulation, the MT was obtained as described by Dessem et al. ${ }^{31}$ In brief, animals were placed in cages with wire mesh flooring and allowed to explore and groom until acclimated. The flexible probe of an electronic von Frey aesthesiometer (Model 2390, IITC/Life Science, Victory Blvd Woodland Hills, CA, USA) was applied to the plantar surface of the hind paw. The MT was automatically recorded when the rat hind paw either abruptly withdrew or made a flinching movement. For each animal, MT values were measured in triplicate and then averaged.

\section{Thermal hyperalgesia}

Paw withdrawal latency (PWL) to noxious heat stimuli was measured using an apparatus for measuring PWL (Model 336, IITC/Life Science, CA). ${ }^{32}$ In brief, the rat was placed in a Plexiglas chamber with a bottom glass plate positioned above a light box. Radiant heat stimulus was applied by directing a beam of light through a hole in the light box onto the heel of each hind paw through the glass plate. The light beam was turned off when the rat lifted the foot, allowing measurement of PWL, which was defined as the time between when the light beam hit the foot and when the foot was lifted. Each trial was performed in triplicate at $5 \mathrm{~min}$ intervals. A cut-off time of $20 \mathrm{~s}$ was imposed to avoid tissue injury.

\section{Histology}

Samples were fixed at room temperature in $4 \%$ neutral buffered formalin and routinely processed for histology. Serial sections $(7 \mu \mathrm{m})$ of paraffin-embedded tissues were cut. Sections were deparaffinized with xylene, stained with hematoxylin and eosin, and analyzed by an expert histopathologist who was blinded to the study. Endometriosis score was determined on the basis of the semiquantitative histological classification proposed by Keenan et $\mathrm{al}^{33}$. This classification is based on the preservation status of epithelial cells in endometrial explants as follows: wellpreserved epithelial layer $=$ score 3 , moderately preserved epithelium with leukocyte infiltrate $=$ score 2 , poorly preserved epithelium (only occasional epithelial cells) $=$ score 1 , and no epithelium $=$ score zero.

\section{Assessment of vessel number}

For each cyst, three sections were deparaffinized and stained with hematoxylin and eosin. Low magnification $(100 \times)$ was used to review the number of vessels in each section and determine the highest vascular staining regions. The five highest vascular staining regions were selected for high magnification $(400 \times)$ vessel count, and the average was calculated. Quantitative evaluations were performed by a researcher who was blinded to the experiment.

\section{Assessment of MC density}

Sections were deparaffinized in xylene and submerged through a graded series of ethanol for $5 \mathrm{~min}$ in each solution. The sections were kept in water for $5 \mathrm{~min}$, transferred to toluidine blue (89640, Sigma, USA) for $10 \mathrm{~min}$, and blotted carefully. Sections were dehydrated in absolute alcohol of 1 min, cleared in xylene, and mounted on the slide using Eukitt (Bio-Optica, Milan, Italy). Microscopically, MCs appeared purple, while the rest of the section was stained blue. MC count was determined in five fields $(200 \times)$ per section using Axiovision Zeiss (Milan, Italy) microscope. In brief, the degranulated MCs possessed irregular shapes with uneven color and incomplete cell membranes, whereas granulated MCs were round or oval with uniform color and intact cell membranes. Quantitative evaluation was performed by a researcher who was blinded to the origin of the material.

\section{Immunohistochemical staining and}

\section{assessment}

Tissues blocks were prepared and sectioned at $7 \mu \mathrm{m}$, deparaffinized, and rehydrated. Sections from endometrial cysts were then incubated overnight with anti-NGF antibody (1:200, ab6199; Abcam, Cambridge, Britain), anti-COX2 antibody (1:600, ab15191; Abcam), anti-ICAM antibody (1:1000, ab124760; Abcam), and anti-VEGF antibody (1:200, ab46154; Abcam). Sections from DRG were incubated with anti-NGF antibody (1:200, ab6199; Abcam) and anti-TRPV1 antibody (1:1000, ab10296; Abcam). After washing with $1 \times$ PBS, the sections were incubated with secondary antibody. Envision-labeled polymer-alkaline phosphatase rabbit (GK400305/15, Novocastra, UK) and diaminobenzidine (GK346810, Novocastra, UK) were used to visualize the antigen-antibody reaction. The immunohistochemistry (IHC) score was the sum of percentage ( $0-3)$ and intensity scores (0-3). Using a Zeiss microscope, the average IHC score of five different fields at 400x magnification was 
regarded as the molecule expression. All slides were analyzed by two blinded observers.

\section{Determination of TNF- $\alpha$ and histamine concentrations in serum}

The TNF- $\alpha$ and histamine concentrations in serum were measured via ELISA in accordance with the instructions indicated in the TNF- $\alpha$ ELISA kit (RTA00, R\&D Systems, USA) and histamine ELISA kit (LS-F27982-1, LifeSpan Biosciences, Seattle, USA), respectively. The TNF- $\alpha$ and histamine concentrations were measured at $450 \mathrm{~nm}$ using a Varioskan ${ }^{\circledR}$ Flash Spectral Scanning Multimode Reader (Thermo Fisher Science, Waltham, MA, USA).

\section{Statistical analysis}

Data were analyzed using GraphPad Prism 6 (Graph Pad Software) and presented as the mean \pm standard error of the mean (SEM). The groups of animals were compared by one-way ANOVA. The correlation between the number of MCs and pain behavior was determined through Pearson correlation. A $p$-value of less than 0.05 was considered significant.

\section{Results}

\section{Ketotifen treatment suppressed the} development of hyperalgesia in rats with experimentally induced endometriosis

No significant difference was found in the withdrawal thresholds and paw withdrawal latency of the randomly grouped rats both before the induction of endometriosis and 7 days post-surgery. However, at day 14, the MTs and PWL in groups with experimentally induced endometriosis were significantly lower than those in the sham operation group. At day 28 of ketotifen or solvent (CMC-Na) intervention, rats administered with ketotifen ( 1 or $10 \mathrm{mg} / \mathrm{kg} /$ day) presented significantly higher MTs and PWL compared with those that received CMC-Na. Although the high-dose ketotifen group $(10 \mathrm{mg} / \mathrm{kg} /$ day $)$ had higher thresholds than the low-dose group (1 mg/kg/day), no statistically significant difference was observed (Figure 1A and B).

\section{Ketotifen treatment decreased the number of MCs and inhibited its activity of degranulation}

In endometriotic cysts, MCs were mainly distributed in the stroma (Figure 2A-C) and around blood vessels (Figure 2D-F) as visualized by toluidine blue staining. The number of MCs in the ketotifen-treated rats were significantly reduced in both low-dose (Figure 2A and D) and high-dose (Figure 2B and E) groups, when Compared with the control group (Figure 2C and F) ( $p<0.001$; Table 1). Additionally, the degranulation rate in the control group was higher than that in the ketotifentreated groups $(p<0.001$; Table 1$)$. However, no statistically significant difference was noted between the low-dose and high-dose groups $(p>0.05)$.

\section{Ketotifen treatment suppressed the} development of endometriotic lesions in rats with experimentally induced

\section{endometriosis}

All uterine implants displayed transparent cystic areas at 28 days post-surgery. The control (CMC-Na) and ketotifen groups developed cysts in the mesentery and abdominal wall. Histological sections from the ketotifen-treated rats showed marked atrophy and regression of the endometriotic implant epithelium in the low- and high-dose groups (Figure 3A and B). In the control group, cysts were characterized by an intense cellular infiltration and a wellpreserved viable epithelium of the endometriotic implant (Figure 3C) $(p<0.001$, Figure 3G). The number of vessels in the ketotifen-treated groups (Figure $3 \mathrm{D}$ and $\mathrm{E}$ ) was lower than that in the control group (Figure $3 \mathrm{~F}$ ) $(p<0.001$, Figure $3 \mathrm{H})$. No significant difference was found in the low-dose and high-dose groups $(p>0.05)$. In addition, groups did not differ in cyst number, but cyst size was smaller in the ketotifen-treated rats in the low-dose $(1 \mathrm{mg} / \mathrm{kg})$ and high-dose groups $(10 \mathrm{mg} / \mathrm{kg})$ than in the control rats $(p<0.05$, Figure 3I).

\section{Ketotifen treatment downregulated the expression of NFG, Cox-2, ICAM-I, and VEGF in endometriotic cysts}

Similar expression patterns of NGF (Figure 4A-C), Cox-2 (Figure 4D-F), ICAM-1 (Figure 4G-I), and VEGF (Figure 4J-L) proteins were observed in the cytoplasm of endometriotic glandular cells and stromal cells. The IHC scores of NGF, Cox-2, ICAM-1, and VEGF expression in the control group (NGF, 3.17

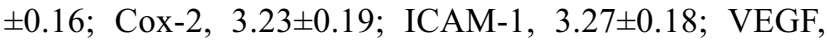
$3.30 \pm 0.20)$ were significantly higher than those in the $1 \mathrm{mg} / \mathrm{kg}$ ketotifen-treated group (NGF, $p<0.001$; Cox-2, $p<0.001$; ICAM-1, $p<0.05$; VEGF, $p<0.05)$ and the 


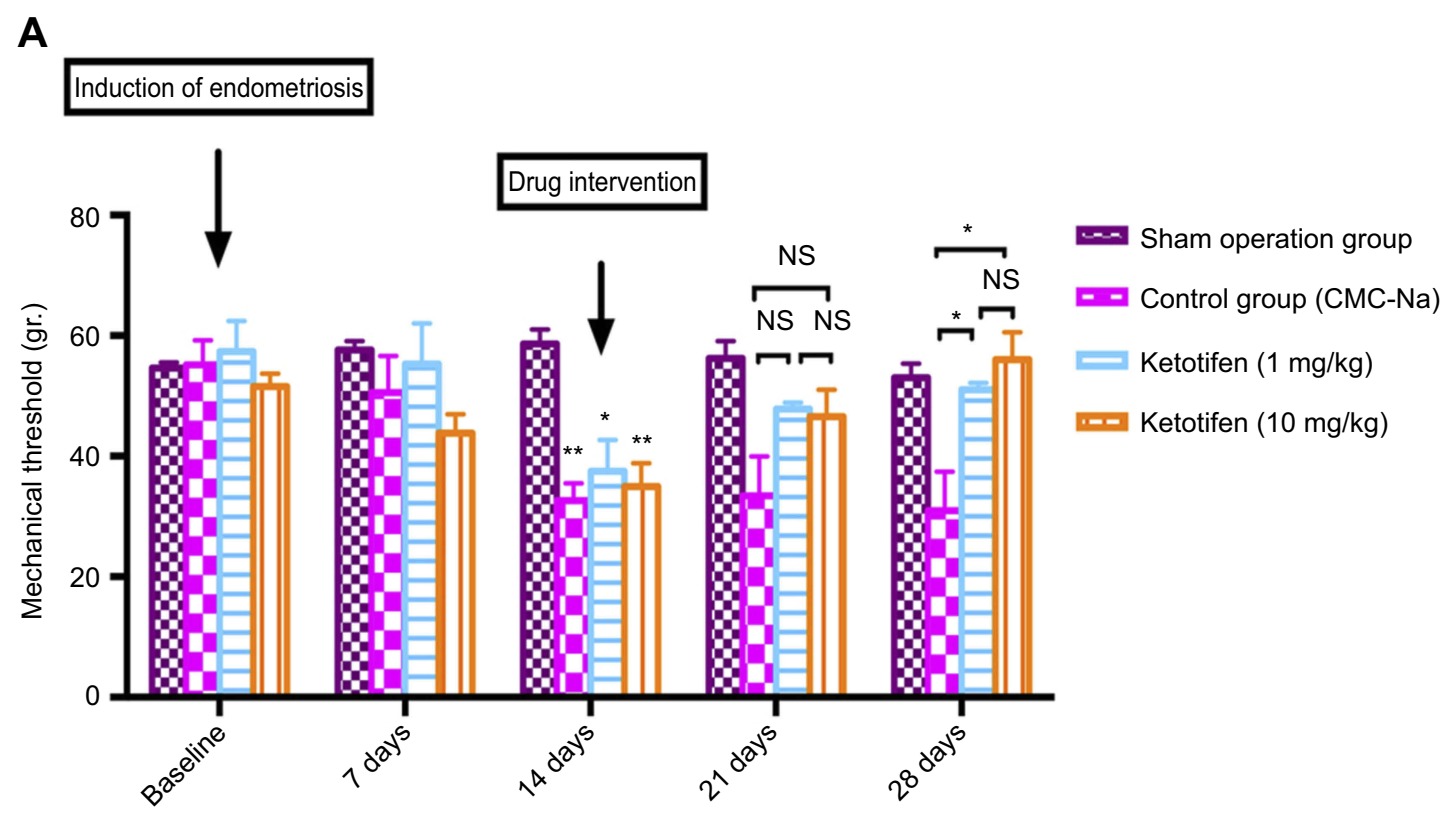

B

Induction of endometriosis

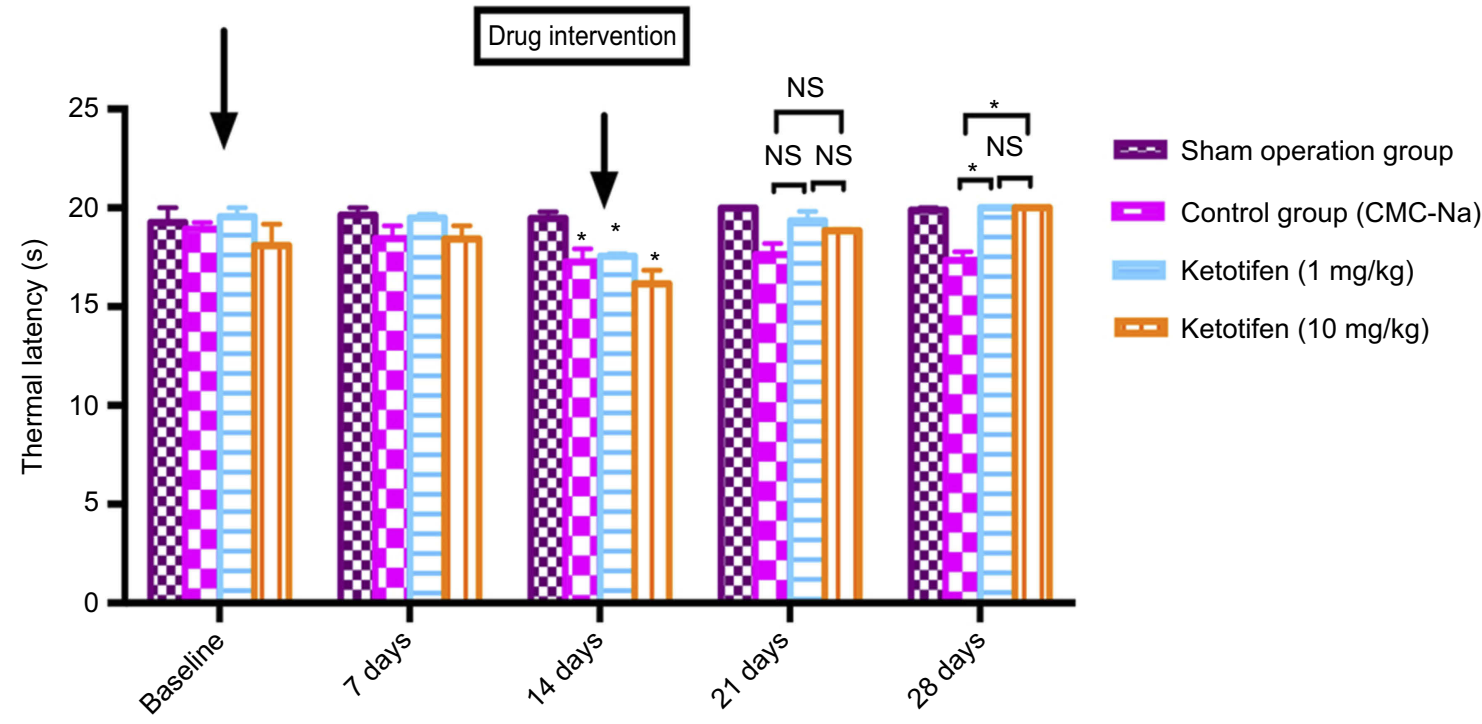

Figure I Ketotifen treatment suppressed the development of hyperalgesia in rats with experimentally induced endometriosis. Mechanical nociceptive testing (A). Thermal nociceptive testing (B). Each group comprised six rats. ${ }^{*} p<0.05$.

Abbreviation: NS, not significant.

$10 \mathrm{mg} / \mathrm{kg}$ ketotifen-treated group (NGF, $p<0.001$; Cox$2, p<0.001$; ICAM-1, $p<0.001$; VEGF, $p<0.05$; Table 2). Moreover, the scores of NGF and ICAM-1 expression were significantly lower in the $10 \mathrm{mg} / \mathrm{kg}$ ketotifentreated group than in the $1 \mathrm{mg} / \mathrm{kg}$ ketotifen-treated group $(p<0.05)$; no significant difference in Cox-2 and VEGF expression was observed in the two dosage groups $(p>0.05)$.

\section{Ketotifen treatment downregulated the} expression of NFG and TRPVI in DRG NGF and TRPV1 staining were both primarily localized in the cytoplasm of DRG cells. Compared with rats in the sham group (Figure 5A and E), increased immunostaining of NFG and TRPV1 was observed in the DRG cells of rats with endometriosis $(p<0.001)$. Treatment with ketotifen resulted in a reduction in immunoreactivity of NGF and TRPV1 in 

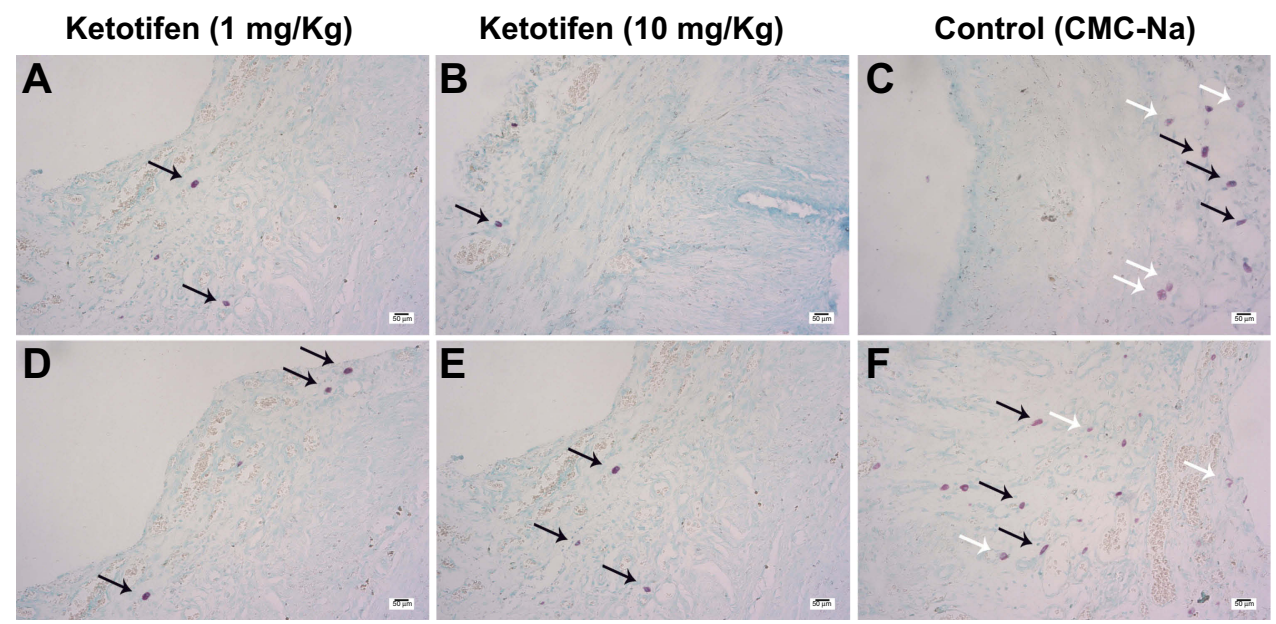

Figure 2 Ketotifen treatment decreased the number of MCs and inhibited its activity of degranulation. MCs were mainly distributed in the stroma (A-C). MCs were noted around blood vessels (D-F). Ketotifen (I/mg/kg/day) (A and D). Ketotifen (I0/mg/kg/day) (B and E). Control (CMC-Na) (C and F). The degranulated MCs, characterized by uneven staining and incomplete cell membranes, were indicated by the white arrows, whereas the granulated MCs, with uniform color and intact cell membranes, were indicated by the black arrow. Scale bars $=50 \mu \mathrm{m}$.

Table I Number of mast cells in endometriotic cysts in rats with or without ketotifen treatment

\begin{tabular}{|l|l|l|l|}
\hline \multirow{2}{*}{$\begin{array}{l}\text { Mast cell } \\
\text { MC) }\end{array}$} & \multicolumn{3}{|l|}{ Group } \\
\cline { 2 - 4 } & $\begin{array}{l}\text { Ketotifen } \\
\text { (I } \mathbf{~ m g / k g )}\end{array}$ & $\begin{array}{l}\text { Ketotifen } \\
\text { (10 } \mathbf{~ m g / k g )}\end{array}$ & $\begin{array}{l}\text { Control } \\
\text { (CMC-Na) }\end{array}$ \\
\hline $\begin{array}{l}\text { Granulated } \\
\text { MC } \\
\text { Degranulated } \\
\text { MC } \\
\begin{array}{l}\text { Total number } \\
\text { of MC }\end{array}\end{array}$ & $3.33 \pm 0.29$ & $3.17 \pm 0.27$ & $4.53 \pm 1.55$ \\
\hline
\end{tabular}

Notes: Values are mean \pm SEM; $* * * p<0.00$ I (compared with control group), $p>0.05$ (Ketotifen $[1 \mathrm{mg} / \mathrm{kg}]$ vs ketotifen $[10 \mathrm{mg} / \mathrm{kg}]$ ).

the DRG of rats with endometriosis in the low-dose (NGF, $p<0.01$, Figure 5B; TRPV1, $p<0.01$, Figure 5F) and highdose groups (NGF, $p<0.001$, Figure 5C; TRPV1, $p<0.001$, Figure 5G) compared with control group (Figure 5D and $\mathrm{H}$ ) (Table 2). Moreover, the $10 \mathrm{mg} / \mathrm{kg}$ ketotifen-treated group had significantly lower TRPV1 expression than the $1 \mathrm{mg} / \mathrm{kg}$ ketotifen-treated group $(p<0.05)$. By contrast, NGF expression did not show a significant difference in these two dosage groups $(p>0.05)$.

\section{Ketotifen treatment reduced histamine and TNF- $\alpha$ concentrations in serum}

Increased concentration of histamine was observed in the serum of rats with endometriosis compared with rats in the sham group, whereas no significant difference was noted in the TNF- $\alpha$ concentration in both groups. Treatment with ketotifen in both low $(1 \mathrm{mg} / \mathrm{kg})$ and high doses $(10 \mathrm{mg} / \mathrm{kg})$ reduced the histamine (Figure 6A) and TNF- $\alpha$ (Figure 6B) concentrations in rats with endometriosis compared with treatment with vehicle $(p<0.05)$. No significant difference in the histamine and TNF- $\alpha$ concentrations was observed in the two ketotifen dosage groups $(p>0.05)$.

\section{Correlation between the number of MCs and pain}

Spearman analysis results showed that the number of total MCs was positively correlated with mechanical hyperalgesia $(\mathrm{r}=-0.47, p<0.05$; Figure $7 \mathrm{~A})$ but not with thermal latency $(\mathrm{r}=-0.20, p>0.05$; Figure $7 \mathrm{~B})$. Moreover, the number of degranulated MCs in endometriotic cysts was positively correlated with mechanical hyperalgesia $(\mathrm{r}=-0.52$, $p<0.05$; Figure $7 \mathrm{C})$ and thermal latency $(\mathrm{r}=-0.61, p<0.01$; Figure 7D).

\section{Discussion}

Several treatments are available for women with endometriosis-associated pain; however, some cases remain problematic as clinically significant results require prolonged administration, which can expose women to side effects. ${ }^{3-5}$ Our study evaluated a new possibility for endometriosis therapy using the MC stabilizer ketotifen in a rodent model of surgically induced endometriosis. Our results showed that oral treatment with ketotifen could significantly suppress the development of hyperalgesia and reduce the histological score and cyst volume in endometriosis-afflicted rats. This 

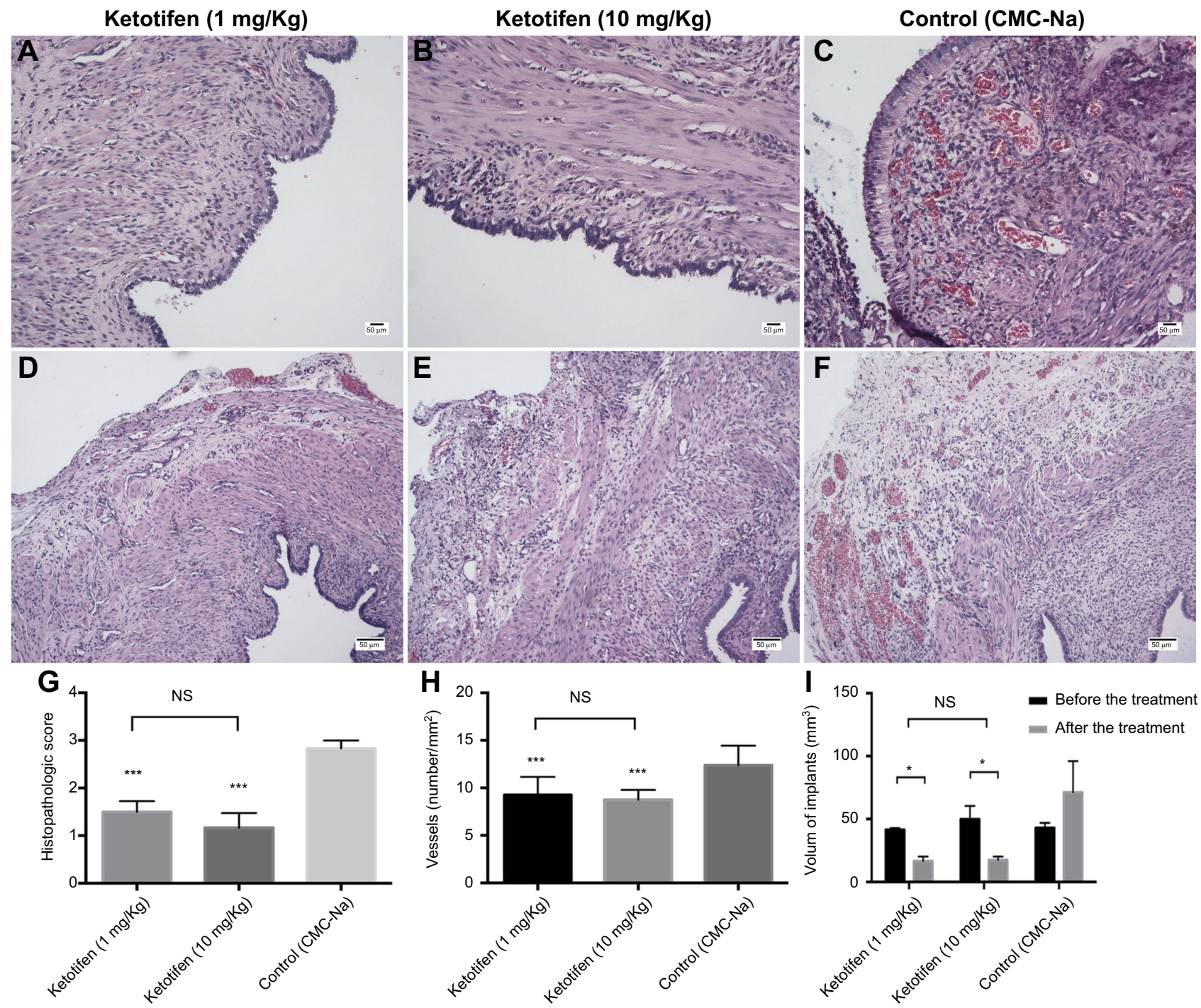

Figure 3 Ketotifen treatment suppressed the development of endometriotic lesions in rats subjected to experimentally induced endometriosis. Histology of tissues (A-C). Density of blood vessels (D-F). Histopathologic scores of different treatments in endometriosis-afflicted rats (G). Density of blood vessels Number of blood vessels in different treatments in endometriosis-afflicted rats $\mathbf{( H )}$. Changes in cyst volume before and after different treatments in endometriosis-afflicted rats $(\mathbf{I})$. Scale bars $=50 \boldsymbol{\mu m}$. $* p<0.05 ; * * * p<0.001$.

positive effect occurred in the absence of any significant adverse event or signs of chronic suffering.

In this study, our data showed that 1 and $10 \mathrm{mg} / \mathrm{kg}$ regimens had significant effects in counteracting pain behavior. A clear dose effect was not observed because no significant difference was found between the two doses in the suppression of hyperalgesia or the reduction in cyst volume in endometriosis-afflicted rats. Our study was the first to evaluate the effects of ketotifen in an animal model of endometriosis, which likely involved altered function of peripheral sensitization sustained by the dysregulation of MC activity. The involvement of MCs in endometriosis is well known, ${ }^{6-10}$ and they are a recognized target for ketotifen action. Histological analysis showed that oral ketotifen treatment significantly reduced the number and activity of MCs in endometriotic cysts. This result was in line with the suppressed development of hyperalgesia through nociceptive testing in our study. MC granules contain pro-algogenic mediators, primarily NGF. ${ }^{34}$ After ketotifen oral treatment, we found a significant reduction in NGF levels in endometriotic cysts and DRG cells.

As previously reported, MCs play a role in angiogenesis, which is a key feature of endometriosis. ${ }^{35}$ For this reason, we first studied the role of ketotifen in angiogenesis during endometriosis and demonstrated its strong anti-angiogenic property. The anti-angiogenic effect of ketotifen was corroborated by immunohistochemical staining of VEGF, which is the main pro-angiogenic mediator in endometriotic cysts. The downregulation of 


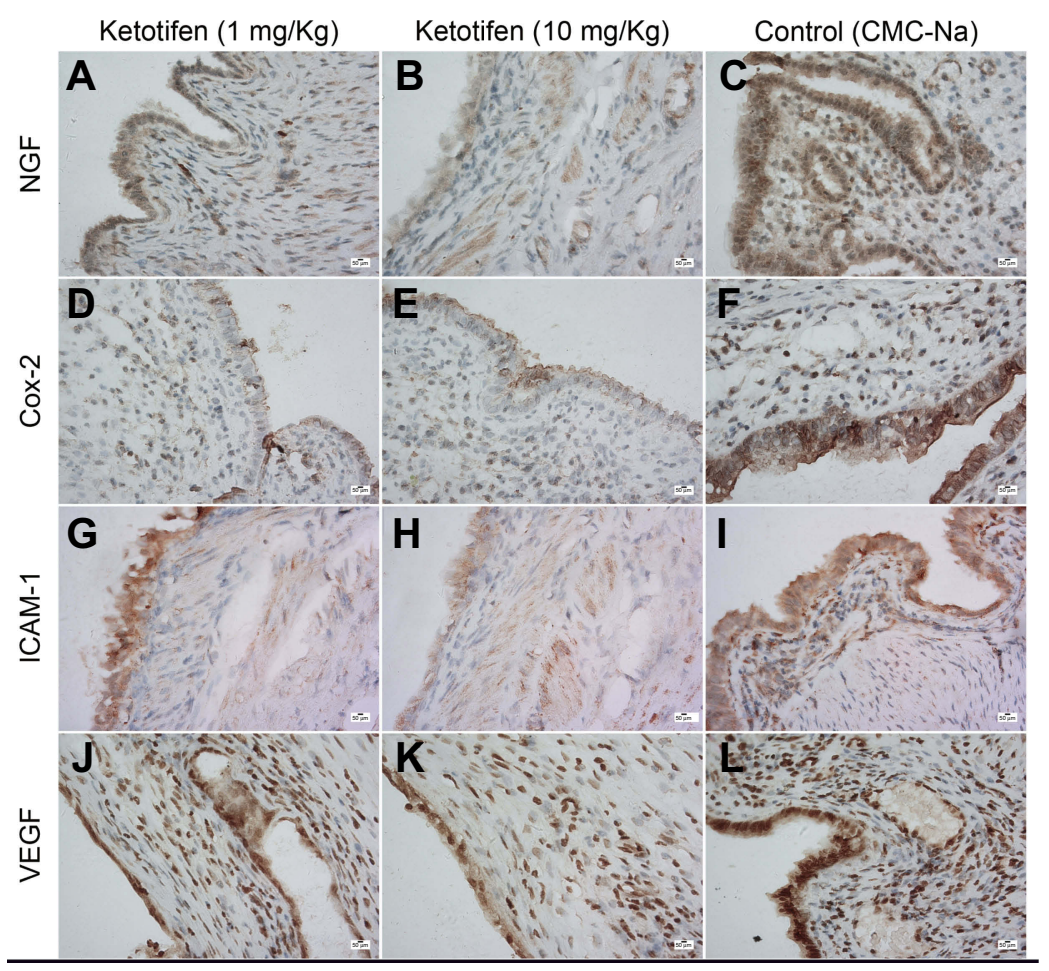

Figure 4 Ketotifen treatment downregulated the expression of NGF, Cox-2, ICAM-I, and VEGF in endometriotic cysts. Scale bars=50 $\mu \mathrm{m}$.

Table 2 Immunohistochemical score of NGF, Cox-2, ICAM-I, and VEGF in endometriotic cysts and NGF and TRPVI in DRG

\begin{tabular}{|c|c|c|c|c|}
\hline \multirow[t]{2}{*}{ IHC Score } & \multicolumn{4}{|l|}{ Groups } \\
\hline & Ketotifen (I mg/kg) & Ketotifen (10 mg/kg) & Control (CMC-Na) & Sham operation \\
\hline \multicolumn{5}{|l|}{ Endometriotic cyst } \\
\hline NGF & $2.40 \pm 0.10 * * *$ & $2.10 \pm 0.10 * * * a$ & $3.17 \pm 0.16$ & NA \\
\hline Cox-2 & $2.37 \pm 0.15^{* * * *}$ & $2.30 \pm 0.14 * * *$ & $3.23 \pm 0.19$ & NA \\
\hline ICAM-I & $2.73 \pm 0.16 *$ & $2.23 \pm 0.77 * * * b$ & $3.27 \pm 0.18$ & NA \\
\hline VEGF & $2.77 \pm 0.20^{*}$ & $2.76 \pm 0.16 *$ & $3.30 \pm 0.20$ & NA \\
\hline \multicolumn{5}{|l|}{ DRG } \\
\hline NGF & $2.14 \pm 0.20 * *$ & $1.96 \pm 0.20 * * *$ & $3.10 \pm 0.19$ & $1.10 \pm 0.14 * * *$ \\
\hline TRPVI & $2.43 \pm 0.18 * * *$ & $1.78 \pm 0.17 * * * c$ & $3.70 \pm 0.22$ & $1.32 \pm 0.17^{* * *}$ \\
\hline
\end{tabular}

Notes: Values are mean \pm SEM. ${ }^{*} p<0.05, * * p<0.01,{ }^{*} *{ }^{*} p<0.001$ (compared with control group); ${ }^{a} p<0.05$ (NGF); ${ }^{b} p<0.05$ (ICAM-I); ${ }^{m} p>0.05$ (VEGF); ${ }^{c} p<0.05$ (TRPVI) (Ketotifen (I mg/kg) vs ketotifen $(10 \mathrm{mg} / \mathrm{kg})$ )

Abbreviations: NGF, nerve growth factor; Cox-2, cyclooxygenase-2; ICAM-I, intercellular cell adhesion molecule-I; VEGF, vascular endothelial growth factor; TRPVI, transient receptor potential cation channel subfamily $\mathrm{V}$ member I; IHC score, immunohistochemical score; DRG, dorsal root ganglia; SEM, standard error of the mean.

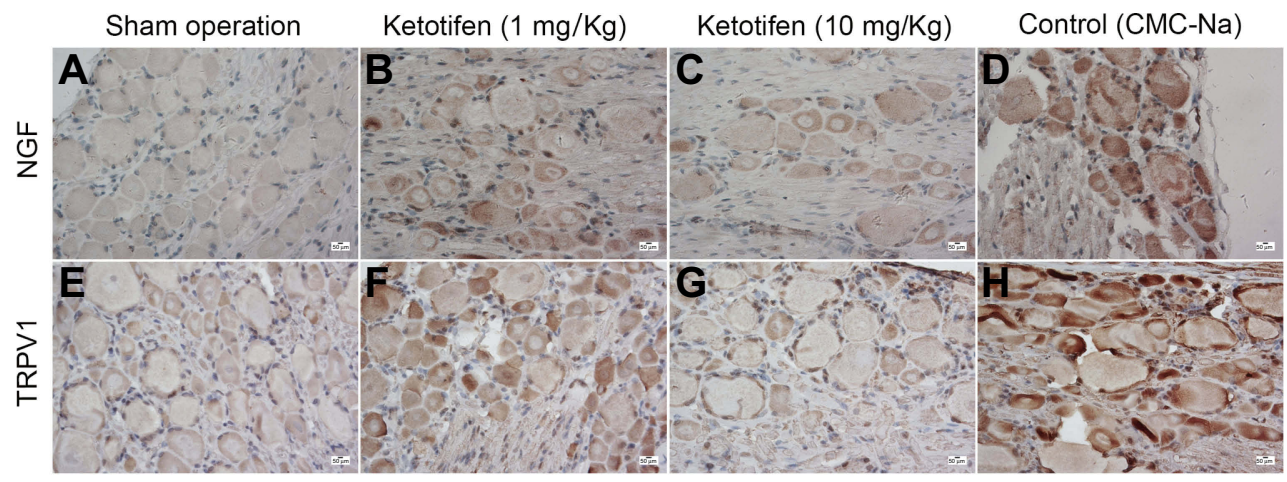

Figure 5 Ketotifen treatment downregulated the expression of NGF and TRPVI in dorsal root ganglia (DRG). Scale bars=50 $\mu \mathrm{m}$. 
A

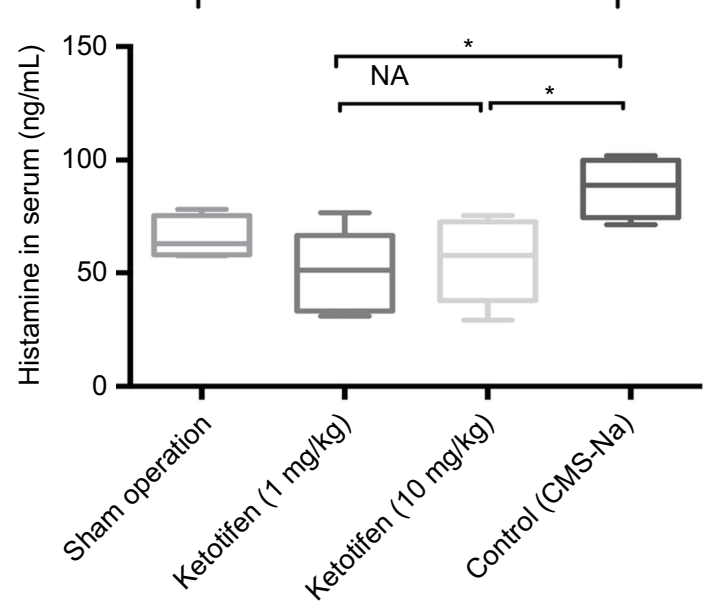

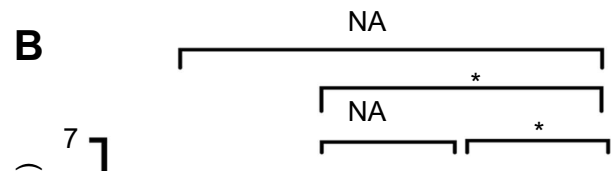

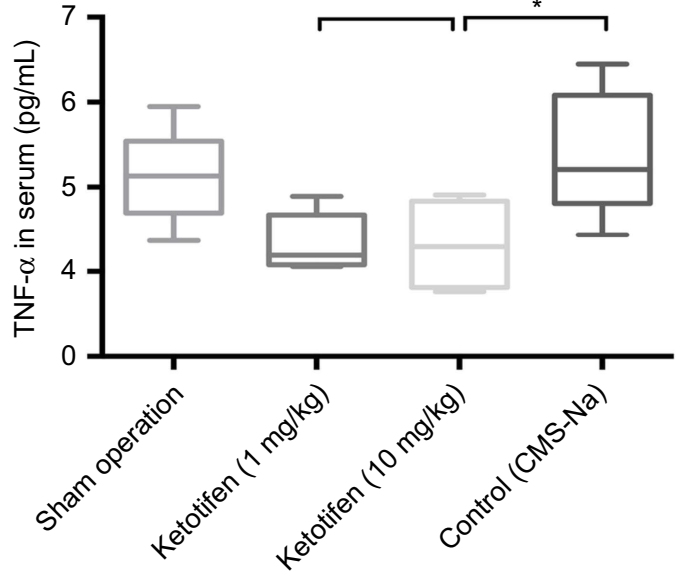

Figure 6 Ketotifen treatment reduced histamine and TNF- $\boldsymbol{\alpha}$ concentrations in serum. Histamine concentration in serum $(\mathbf{A})$. TNF- $\boldsymbol{\alpha}$ concentration in serum $(\mathbf{B}) . * P<0.05$.

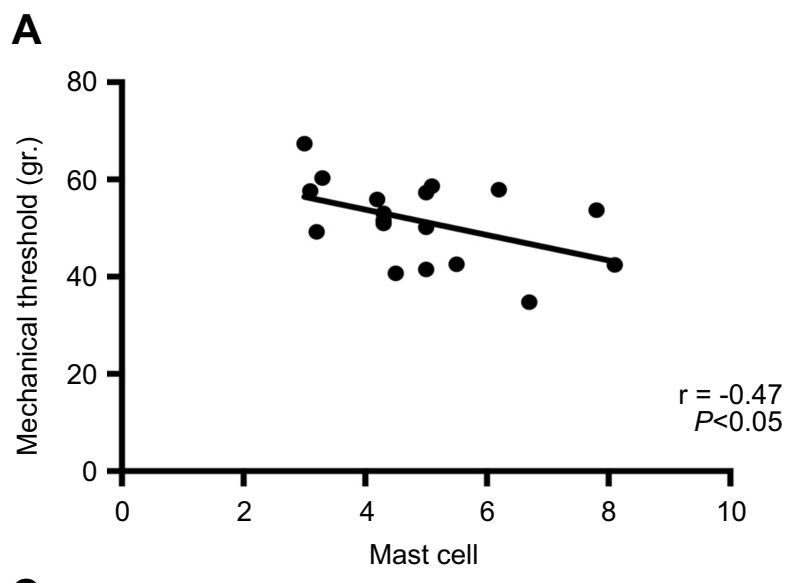

B

C
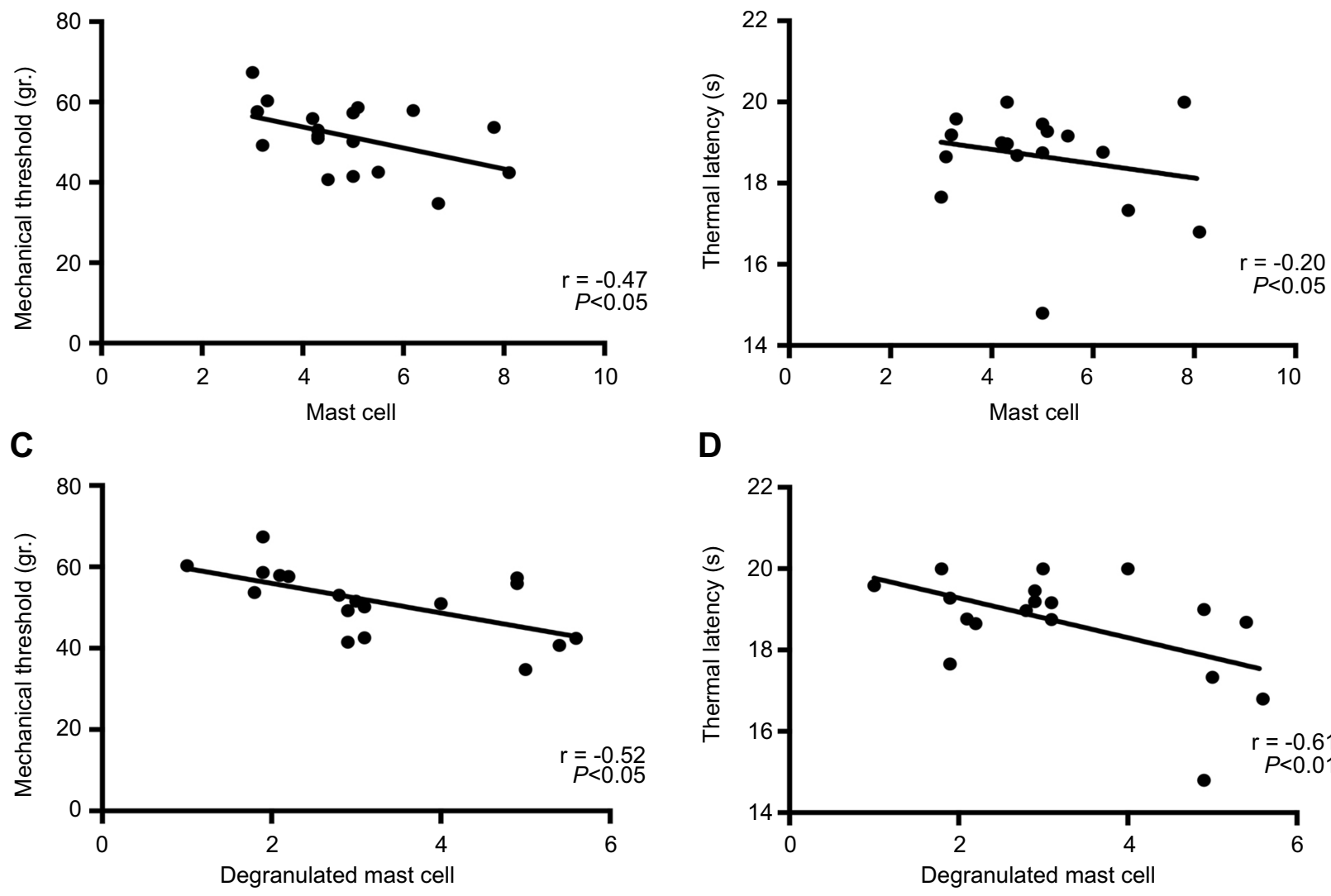

D

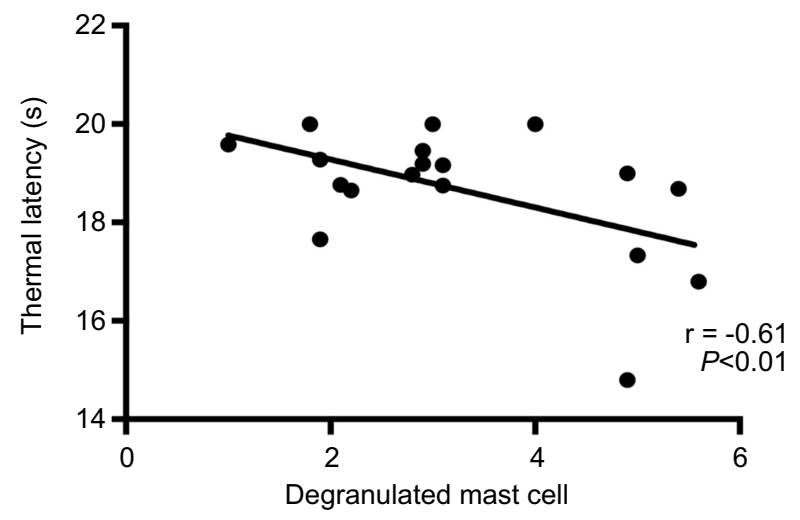

Figure 7 Correlations between the number of $M C$ and hyperalgesia. Correlation of number of $M C$ and mechanical threshold (A) and thermal latency (B); Correlation of number of degranulated MC and mechanical threshold $(\mathbf{C})$ and thermal latency (D). The Pearson coefficient of correlation and the significance of this correlation are shown in the lower right corner.

VEGF was likely due to the MC modulation exerted by ketotifen, because VEGF is released mainly by MCs during chronic inflammation. ${ }^{36}$ Moreover, the reduction in angiogenesis associated with ketotifen treatment may partly justify the reduction in cyst diameter reported in this paper.

An increasing body of evidence indicated that ketotifen has an anti-inflammatory effect in many pathological 
conditions. $^{28,37}$ This phenomenon was proven by our results. ICAM-1 has a crucial role in endometriosis. ${ }^{38}$ Treatment with ketotifen reduced ICAM-1 upregulation, thereby decreasing the interaction between neutrophil and endothelial cells during adhesion.

Biochemical analysis of DRG showed that oral ketotifen treatment reduced NGF protein expression at this level. This result strongly supported the notion that ketotifen exerted its anti-algogenic effect by modulating NGF, one of the main mediators activating the algogenic input from the endometriosis lesions toward the nervous system. ${ }^{39}$ Retrograde transport of NGF released from inflamed tissue acts to increase translation and transport of TRPV1 to the peripheral nociceptor terminal, where it contributes to the maintenance of inflammatory pain hypersensitivity. ${ }^{40}$ Our results showed that oral treatment also reduced TRPV1 repression in DRG cells, which may result from ketotifen blocking NGF release by MCs, since previous studies have reported that MCs can synthesize, store, and release NGF. ${ }^{41}$

Our results also showed that endometriosis-afflicted rats treated with ketotifen had reduced histamine and TNF- $\alpha$ concentrations in serum compared with placebotreated rats. In view of these findings, our data on reduced pain behavior could be largely attributed to the reduction in the amount of algogenic mediators produced by MCs during endometriosis and the consequent degree of peripheral sensitization due to ketotifen administration.

Regardless of possible mechanisms, our findings suggest that orally administered ketotifen in patients with endometriosis with pain could be particularly useful, whether alone or in combination with classic anti-inflammatory/analgesic treatments. This drug is mainly used in patients with asthma and other allergic diseases, and its safety has already been confirmed, which is a very important advantage before clinical application. Further experimental and clinical studies will be needed for confirmation because no clear dose-effect relationship was observed in our experiments.

\section{Acknowledgments}

We thank Cai-Yun Zhou and Ming-Hua Yu for their help with quantification of immunohistochemistry, and HaoHao Lu for his help in animal modeling and drug administration. This work was supported by the National Key R\&D Program of China (grant number 2017YFC1001202) and the National Natural Science Foundation of China (grant numbers 81471433, 81671429, 81471127).

\section{Disclosure}

The authors declare that there are no conflicts of interest that could be perceived as prejudicing the impartiality of the research reported.

\section{References}

1. Strathy JH, Molgaard CA, Coulam CB, Melton LJ 3rd Endometriosis and infertility: a laparoscopic study of endometriosis among fertile and infertile women. Fertil Steril. 1982;38(6):667-672.

2. Kennedy S, Bergqvist A, Chapron C, et al. ESHRE guideline for the diagnosis and treatment of endometriosis. Hum Reprod. 2005;20 (10):2698-2704. doi:10.1093/humrep/dei135

3. Rostom A, Dube C, Wells G, et al. Prevention of NSAID-induced gastroduodenal ulcers. Cochrane Database Syst Rev. 2002;(4):CD002296.

4. Gaytan M, Morales C, Bellido C, Sanchez-Criado JE, Gaytan F. Nonsteroidal anti-inflammatory drugs (NSAIDs) and ovulation: lessons from morphology. Histol Histopathol. 2006;21(5):541-556. doi:10.14670/HH-21.541

5. Pierce SJ, Gazvani MR, Farquharson RG. Long-term use of gonadotropin-releasing hormone analogs and hormone replacement therapy in the management of endometriosis: a randomized trial with a 6-year follow-up. Fertil Steril. 2000;74(5):964-968.

6. Anaf V, Chapron C, El Nakadi I, De Moor V, Simonart T, Noel JC. Pain, mast cells, and nerves in peritoneal, ovarian, and deep infiltrating endometriosis. Fertil Steril. 2006;86(5):1336-1343. doi:10.1016/ j.fertnstert.2006.03.057

7. Fujiwara H, Konno R, Netsu S, et al. Localization of mast cells in endometrial cysts. Am J Reprod Immunol. 2004;51(5):341-344. doi:10.1111/j.1600-0897.2004.00166.x

8. Kirchhoff D, Kaulfuss S, Fuhrmann U, Maurer M, Zollner TM. Mast cells in endometriosis: guilty or innocent bystanders? Expert Opin Ther Targets. 2012;16(3):237-241. doi:10.1517/14728222.2012.661415

9. Matsuzaki S, Canis M, Darcha C, Fukaya T, Yajima A, Bruhat MA. Increased mast cell density in peritoneal endometriosis compared with eutopic endometrium with endometriosis. Am $J$ Reprod Immunol. 1998;40(4):291-294.

10. Sugamata M, Ihara T, Uchiide I. Increase of activated mast cells in human endometriosis. Am J Reprod Immunol. 2005;53(3):120-125. doi:10.1111/j.1600-0897.2005.00254.x

11. Aich A, Afrin LB, Gupta K. Mast cell-mediated mechanisms of nociception. Int J Mol Sci. 2015;16(12):29069-29092. doi:10.3390/ ijms161226151

12. Cenac N, Altier C, Motta JP, et al. Potentiation of TRPV4 signalling by histamine and serotonin: an important mechanism for visceral hypersensitivity. Gut. 2010;59(4):481-488. doi:10.1136/gut.2009.192567

13. Rudick CN, Bryce PJ, Guichelaar LA, Berry RE, Klumpp DJ. Mast cell-derived histamine mediates cystitis pain. PLoS One. 2008;3:5. doi:10.1371/journal.pone.0002096

14. Sorkin LS, Xiao WH, Wagner R, Myers RR. Tumour necrosis factor-alpha induces ectopic activity in nociceptive primary afferent fibres. Neuroscience. 1997;81(1):255-262.

15. Biedermann T, Kneilling M, Mailhammer R, et al. Mast cells control neutrophil recruitment during T cell-mediated delayed-type hypersensitivity reactions through tumor necrosis factor and macrophage inflammatory protein 2. J Exp Med. 2000;192(10):1441-1452.

16. Zhu LB, Lin KQ, Zhang XM, Lin J. [Sodium cromoglycate attenuates experimental endometriosis in rats by regulating mast cells]. Zhejiang Da Xue Xue Bao Yi Xue Ban. 2015;44(3):278-284.

17. Phillips MJ, Meyrick Thomas RH, Moodley I, Davies RJ. A comparison of the in vivo effects of ketotifen, clemastine, chlorpheniramine and sodium cromoglycate on histamine and allergen induced weals in human skin. Br J Clin Pharmacol. 1983;15 (3):277-286. 
18. Martin U, Roemer D. Ketotifen: a histamine release inhibitor. Monogr Allergy. 1977;12:145-149.

19. Craps LP, Ney UM. Ketotifen: current views on its mechanism of action and their therapeutic implications. Respiration. 1984;45 (4):411-421. doi:10.1159/000194648

20. Sokol KC, Amar NK, Starkey J, Grant JA. Ketotifen in the management of chronic urticaria: resurrection of an old drug. Ann Allergy Asthma Immunol. 2013;111(6):433-436. doi:10.1016/j.anai.2013.10.003

21. Barreau F, Salvador-Cartier C, Houdeau E, Bueno L, Fioramonti J. Long-term alterations of colonic nerve-mast cell interactions induced by neonatal maternal deprivation in rats. Gut. 2008;57(5):582-590. doi:10.1136/gut.2007.126680

22. Song J, Zhang L, Bai T, Qian W, Li R, Hou X. Mast cell-dependent mesenteric afferent activation by mucosal supernatant from different bowel segments of guinea pigs with post-infectious irritable bowel syndrome. J Neurogastroenterol Motil. 2015;21(2):236-246. doi:10.5056/jnm14095

23. Ang DC, Hilligoss J, Stump T. Mast cell stabilizer (Ketotifen) in fibromyalgia: phase 1 randomized controlled clinical trial. Clin J Pain. 2014. doi:10.1097/AJP.0000000000000169

24. Blanco I, Beritze N, Arguelles M, et al. Abnormal overexpression of mastocytes in skin biopsies of fibromyalgia patients. Clin Rheumatol. 2010;29(12):1403-1412. doi:10.1007/s10067-010-1474-7

25. Oliveira SM, Drewes CC, Silva CR, et al. Involvement of mast cells in a mouse model of postoperative pain. Eur J Pharmacol. 2011;672 (1-3):88-95. doi:10.1016/j.ejphar.2011.10.001

26. Oliveira SM, Silva CR, Ferreira J. Critical role of protease-activated receptor 2 activation by mast cell tryptase in the development of postoperative pain. Anesthesiology. 2013;118(3):679-690. doi:10.1097/ ALN.0b013e31827d415f

27. Rajkumar K, Schott PW, Simpson CW. The rat as an animal model for endometriosis to examine recurrence of ectopic endometrial tissue after regression. Fertil Steril. 1990;53(5):921-925.

28. Anoush M, Mohammad Khani MR. Evaluating the anti-nociceptive and anti-inflammatory effects of ketotifen and fexofenadine in rats. Adv Pharm Bull. 2015;5(2):217-222. doi:10.15171/apb.2015.030

29. Pothoulakis C, Karmeli F, Kelly CP, et al. Ketotifen inhibits clostridium difficile toxin A-induced enteritis in rat ileum. Gastroenterology. 1993;105(3):701-707.
30. Ozcan Cenksoy P, Oktem M, Erdem O, et al. A potential novel treatment strategy: inhibition of angiogenesis and inflammation by resveratrol for regression of endometriosis in an experimental rat model. Gynecol Endocrinol. 2015;31(3):219-224. doi:10.3109/ 09513590.2014 .976197

31. Dessem D, Ambalavanar R, Evancho M, Moutanni A, Yallampalli C, Bai G. Eccentric muscle contraction and stretching evoke mechanical hyperalgesia and modulate CGRP and P2X(3) expression in a functionally relevant manner. Pain. 2010;149(2):284-295. doi:10.1016/j.pain.2010.02.022

32. Hargreaves K, Dubner R, Brown F, Flores C, Joris J. A new and sensitive method for measuring thermal nociception in cutaneous hyperalgesia. Pain. 1988;32(1):77-88.

33. Keenan JA, Williams-Boyce PK, Massey PJ, Chen TT, Caudle MR, Bukovsky A. Regression of endometrial explants in a rat model of endometriosis treated with the immune modulators loxoribine and levamisole. Fertil Steril. 1999;72(1):135-141.

34. Kritas SK, Caraffa A, Antinolfi P, et al. Nerve growth factor interactions with mast cells. Int J Immunopathol Pharmacol. 2014;27 (1):15-19. doi:10.1177/039463201402700103

35. Groothuis PG, Nap AW, Winterhager E, Grummer R. Vascular development in endometriosis. Angiogenesis. 2005;8(2):147-156. doi:10.1007/ s10456-005-9005-x

36. Genovese A, Detoraki A, Granata F, Galdiero MR, Spadaro G, Marone G. Angiogenesis, lymphangiogenesis and atopic dermatitis. Chem Immunol Allergy. 2012;96:50-60. doi:10.1159/000331883

37. Hsu DZ, Chu PY, Chen SJ, Liu MY. Mast cell stabilizer ketotifen inhibits gouty inflammation in rats. Am J Ther. 2016;23(4):e1009e1015. doi:10.1097/MJT.0b013e31829ea238

38. Mousa SA. Cell adhesion molecules: potential therapeutic and diagnostic implications. Methods Mol Med. 2004;93:157-174.

39. Shu XQ, Mendell LM. Neurotrophins and hyperalgesia. Proc Natl Acad Sci USA. 1999;96(14):7693-7696.

40. Ji RR, Samad TA, Jin SX, Schmoll R, Woolf CJ. p38 MAPK activation by NGF in primary sensory neurons after inflammation increases TRPV1 levels and maintains heat hyperalgesia. Neuron. 2002;36(1):57-68.

41. Leon A, Buriani A, Dal Toso R, et al. Mast cells synthesize, store, and release nerve growth factor. Proc Natl Acad Sci U S A. 1994;91(9):3739_ 3743 .
Journal of Pain Research

\section{Publish your work in this journal}

The Journal of Pain Research is an international, peer reviewed, open access, online journal that welcomes laboratory and clinical findings in the fields of pain research and the prevention and management of pain Original research, reviews, symposium reports, hypothesis formation and commentaries are all considered for publication. The manuscript management system is completely online and includes a very quick and fair peer-review system, which is all easy to use. Visit http:// www.dovepress.com/testimonials.php to read real quotes from published authors. 